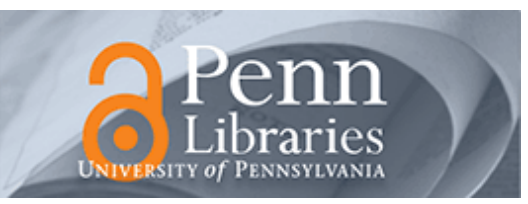

University of Pennsylvania

ScholarlyCommons

April 2003

\title{
A New Approach for Near-Field Wideband Synthetic Aperture Beamforming
}

\author{
Fauzia Ahmad \\ Villanova University \\ Gordon J. Frazer \\ Defence Science and Technology Organisation
}

Saleem A. Kassam

University of Pennsylvania, kassam@seas.upenn.edu

Moeness G. Amin

Villanova University

Follow this and additional works at: https://repository.upenn.edu/ese_papers

\section{Recommended Citation}

Fauzia Ahmad, Gordon J. Frazer, Saleem A. Kassam, and Moeness G. Amin, "A New Approach for NearField Wideband Synthetic Aperture Beamforming", . April 2003.

Copyright 2003 IEEE. Reprinted from Proceedings of the IEEE International Conference on Acoustics, Speech, and Signal Processing 2003 (ICASSP 2003), Volume 5, pages 89-92.

Publisher URL: http://ieeexplore.ieee.org/xpl/tocresult.jsp?isNumber=26996\&page=1

This material is posted here with permission of the IEEE. Such permission of the IEEE does not in any way imply IEEE endorsement of any of the University of Pennsylvania's products or services. Internal or personal use of this material is permitted. However, permission to reprint/republish this material for advertising or promotional purposes or for creating new collective works for resale or redistribution must be obtained from the IEEE by writing to pubs-permissions@ieee.org. By choosing to view this document, you agree to all provisions of the copyright laws protecting it.

This paper is posted at ScholarlyCommons. https://repository.upenn.edu/ese_papers/39

For more information, please contact repository@pobox.upenn.edu. 


\title{
A New Approach for Near-Field Wideband Synthetic Aperture Beamforming
}

\author{
Abstract \\ A coarray-based synthetic aperture beamformer using stepped-frequency signal synthesis and post-data \\ acquisition processing is presented for wideband imaging of near-field scenes. The proposed \\ beamformer formulation and implementation finds key applications in through-the-wall microwave \\ imaging and landmine detection problems. While coarray techniques offer significant reduction in array \\ elements for a given angular resolution, stepped-frequency realization of wideband systems simplifies \\ implementation and offers flexibility in beamforming. Proof of concept is provided using real data \\ collected in an anechoic chamber.

\section{Comments} \\ Copyright 2003 IEEE. Reprinted from Proceedings of the IEEE International Conference on Acoustics, \\ Speech, and Signal Processing 2003 (ICASSP 2003), Volume 5, pages 89-92. \\ Publisher URL: http://ieeexplore.iee. $\mathrm{org} / \mathrm{xpl} /$ tocresult.jsp?isNumber=26996\&page=1 \\ This material is posted here with permission of the IEEE. Such permission of the IEEE does not in any way \\ imply IEEE endorsement of any of the University of Pennsylvania's products or services. Internal or \\ personal use of this material is permitted. However, permission to reprint/republish this material for \\ advertising or promotional purposes or for creating new collective works for resale or redistribution must \\ be obtained from the IEEE by writing to pubs-permissions@ieee.org. By choosing to view this document, \\ you agree to all provisions of the copyright laws protecting it.
}




\title{
A NEW APPROACH FOR NEAR-FIELD WIDEBAND SYNTHETIC APERTURE BEAMFORMING ${ }^{*}$
}

\author{
Fauzia Ahmad ${ }^{1}$, Gordon J. Frazer ${ }^{2}$, Saleem A. Kassam ${ }^{3}$, and Moeness G. Amin ${ }^{1}$ \\ ${ }^{1}$ Center for Advanced Communications, \\ Villanova University, \\ Villanova, PA 19085. \\ e-mail: fahmad@ece.vill.edu; amin@ece.vill.edu \\ ${ }^{2}$ ISR Division, DSTO, \\ Edinburgh, Australia. \\ e-mail: frazer@ieee.org \\ ${ }^{3}$ Dept. of Electrical Engineering, \\ University of Pennsylvania, \\ Philadelphia, PA 19104. \\ e-mail: kassam@ee.upenn.edu
}

\begin{abstract}
A coarray-based synthetic aperture beamformer using stepped-frequency signal synthesis and post-data acquisition processing is presented for wideband imaging of near-field scenes. The proposed beamformer formulation and implementation finds key applications in through-the-wall microwave imaging and landmine detection problems. While coarray techniques offer significant reduction in array elements for a given angular resolution, stepped-frequency realization of wideband systems simplifies implementation and offers flexibility in beamforming. Proof of concept is provided using real data collected in an anechoic chamber.
\end{abstract}

\section{INTRODUCTION}

- Spatial arrays of sensor elements are often used to image sources of reflected power in radar, sonar, and ultrasound applications. Recently, the concept of coarrays was proposed in the literature $[1,2]$, which facilitates addressing fundamental array signal processing issues in a simplified context. The sum coarray was originally defined for narrowband, far-field imaging in [1] and is represented by a set of pair-wise vector sums of the transmit and receive array element locations. This concept was extended to wideband imaging in [3], where it was shown that wideband operation adds extra points at specific locations in the sum coarray. This property can be utilized to enhance the angular resolution achievable with a limited number of array elements.

An aperture synthesis technique using subarrays, was first proposed in [4] for ultrasound applications. In this

* This work was supported by DARPA under Grant No. MDA972-02-1-0022. The content of the information does not necessarily reflect the position or the policy of the Government, and no official endorsement should be inferred. scheme, the transmit and receive arrays are divided into subarray pairs, where each subarray consists of a single transmitter and one or more receivers. These subarrays are used independently to form component complex images of the scene being imaged by post-data acquisition beamforming [4]. These component images are then added coherently to obtain the composite complex amplitude image with the desired spatial resolution. This scheme was later generalized in [5] to incorporate subarrays composed of multiple transmitters using the concept of sum coarray. Until recently, the use of this aperture synthesis scheme was restricted to ultrasound imaging only. It was simultaneously extended to microwave imaging by our group [6], and by a group at University of Bristol, UK [7]. Both these systems use wideband pulses for imaging and divide the transmit and receive arrays into single transmitter/single receiver subarray pairs. All the elements of the intended transmit and receive arrays have to be physically present, and share a pair of processing channels via a subarray multiplexer. Although the work in [7] uses single transmitter/single receiver pairs, our work not only provides a general framework for array synthesis, but also gives us greater control over the sidelobe structure of the point spread function using coarray-based design of array apodizations [5]. This aperture synthesis scheme does not readily suggest itself without considering the concept of sum coarrays. In this paper, we report on important advances made by the authors beyond the previously reported work in [6].

The imaging system described herein makes use of an alternative implementation of the aperture synthesis scheme in which a single subarray, consisting of one transmitter and one receiver, is used. The full transmit and receive array is realized by moving this subarray to different locations forming the array aperture. This reduces the cost of the system further since only two antenna elements, and a pair of transmit and receive processing apparatus is required. The resolution of the 
larger array can thus be obtained with significant reduction in cost at the expense of increased data acquisition time.

Moreover, instead of using pulses, we synthesize a wideband signal using the stepped frequency approach and use this synthesized signal along with post-data acquisition processing to implement a near-field synthetic aperture beamformer in the frequency-domain. It is shown that the application of the stepped-frequency approach leads to a flexible and simplified implementation of the beamformer.

This paper is organized as follows. In Section 2, we describe the post-data acquisition processing and introduce the stepped frequency synthesis of the wideband signal. The frequency domain implementation of the nearfield beamformer is also discussed. Results using real data, demonstrating the working of the wideband, near-field beamformer, are given in Section 3.

\section{DESIGN OF THE SYNTHETIC APERTURE BEAMFORMER}

We describe the design of the near-field, wideband synthetic aperture beamformer in terms of the post-data acquisition processing for aperture synthesis, and the stepped frequency synthesis of the wideband signal.

\subsection{Post-data acquisition processing}

Consider a single transmitter and a single receiver subarray, which is to be used to synthesize an $M$-element transmit and an $N$-element receive line array, both located along the $\mathrm{x}$-axis. The region to be imaged is located along the positive z-axis. Let the transmitter, placed at the $m$-th transmit location, illuminate the scene with a wideband signal $s(t)$. The reflections by targets are measured and stored by the receiver at the $n$-th receive location. For the case of single point target located at $x_{p}=\left(x_{p}, z_{p}\right)$, the output of the $n$-th receiver is given by $a\left(\mathbf{x}_{\mathbf{p}}\right) s\left(t-\tau_{m n}\right)$, where $a\left(\mathbf{x}_{\mathbf{p}}\right)$ is the complex target reflectivity, and $\tau_{m n}$ is the propagation delay encountered by the signal as it travels from the $m$-th transmitter to the target located at $\mathbf{x}_{\mathbf{p}}$, and back to the $n$-th receiver, and is given by

$$
\tau_{m n}=\frac{d\left(\mathbf{x}_{\mathbf{t m}}, \mathbf{x}_{\mathbf{p}}\right)}{c}+\frac{d\left(\mathbf{x}_{\mathbf{p}}, \mathbf{x}_{\mathbf{r n}}\right)}{c}
$$

where $d(\cdot, \cdot)$ is the cartesian distance between two position vectors, $\mathbf{x}_{\mathbf{t m}}$ and $\mathbf{x}_{\mathrm{rn}}$ are the positions vectors of the $m$-th transmit and $n$-th receive locations respectively, and $c$ is the speed of light. This process is repeated, with the transmitter at the $m$-th location, until all the $N$ receive locations have been used sequentially. The $N$ outputs, thus obtained, are processed as follows.

The region of interest is divided into a finite number of pixels in range and angle. The complex composite signal corresponding to the image of the q-th pixel, located at $\mathbf{x}_{\mathbf{q}}$ (at range $\mathrm{R}$ in the direction $\theta$ ), is obtained by applying time delays and weights to the outputs of the $\mathrm{N}$ receivers, and adding them. The result is

$$
z_{m}(t)=\sum_{n=1}^{N} w_{m n} a\left(\mathbf{x}_{\mathbf{p}}\right) s\left(t-\tau_{m n}-\tilde{\tau}_{m n}\right)
$$

where $\tilde{\tau}_{m n}$ is the focusing delay applied to the output of the $n$-th receiver when the transmitter is at the $m$-th location, and is given by

$$
\tilde{\tau}_{m n}=\frac{2 R}{c}-\frac{d\left(\mathbf{x}_{\mathbf{t m}}, \mathbf{x}_{\mathbf{q}}\right)}{c}-\frac{d\left(\mathbf{x}_{\mathbf{q}}, \mathbf{x}_{\mathbf{r n}}\right)}{c}
$$

The component complex image value for the q-th pixel is given by

$$
I_{m}\left(\mathbf{x}_{\mathbf{q}}\right)=\left.z_{m}(t)\right|_{t=\frac{2 R}{c}}
$$

where the sampling time $2 \mathrm{R} / \mathrm{c}$ corresponds to the time of flight of the wideband waveform for the focused range $\mathrm{R}$.

This process is repeated by sequential use of the $M$ transmit locations, one by one. The final complex amplitude image value $I\left(\mathbf{x}_{\mathbf{q}}\right)$ is obtained by the coherent weighted linear combination

$$
I\left(\mathbf{x}_{\mathbf{q}}\right)=\sum_{m=1}^{M} w_{t m} I_{m}\left(\mathbf{x}_{\mathbf{q}}\right)
$$

This process is performed for all pixels to generate the composite image of the region of interest. The general case of multiple targets can be obtained by superposition.

Note that at no time, a formed beam has to be transmitted. Rather, the effect of a focused transmit beam is created by this post-data acquisition processing.

\subsection{Stepped-frequency synthesis}

Frequency-domain representation of the underlying problem is obtained by combining eqs. (2), (4), and (5),

$$
I\left(\mathbf{x}_{\mathbf{q}}\right)=\frac{1}{2 \pi} \int_{-\infty}^{\infty} S(\omega) a\left(\mathbf{x}_{\mathbf{p}}\right) \sum_{m=1}^{M} \sum_{n=1}^{N} w_{t m} w_{m} \exp \left(-j \omega\left(\tau_{m n}+\widetilde{\tau}_{m n}-T\right)\right) d \omega
$$

where $S(\omega)$ is the Fourier transform of $s(t)$ and $T=2 R / c$. The time-delay $\tau_{m n}+\tilde{\tau}_{m n}$ now appears as a frequency dependent phase delay [8]. This implies that beamforming can be achieved by transmitting monochromatic signals corresponding to the continuum of frequencies constituting $S(\omega)$, measuring the complex amplitude of the returns, performing synthetic focusing in the frequency domain for each frequency using a phase delay corresponding to that frequency, and then combining the results per eq. (6).

A stepped-frequency approximation to the above approach would use a finite number, $K$, of monochromatic signals with equi-spaced frequencies covering the desired bandwidth. Equation (6) then becomes

$I\left(\mathbf{x}_{\mathbf{q}}\right)=\frac{1}{2 \pi} \sum_{k=0}^{K-1} S\left(\omega_{k}\right) a\left(\mathbf{x}_{\mathbf{p}}\right) \sum_{m=1}^{M} \sum_{n=1}^{N} w_{t m} w_{r n} \exp \left(-j \omega_{k}\left(\tau_{m n}+\tilde{\tau}_{m n}-T\right)\right)$

where 


$$
\Delta \omega=\left(\omega_{K-1}-\omega_{0}\right) / K ; \omega_{k}=\omega_{0}+k \Delta \omega \text { for } k=0, \cdots, K-1
$$

The difference $\omega_{k \cdot l}-\omega_{b}$ is the desired bandwidth and $\Delta \omega$ is the frequency step size.

Since we transmit and receive $\mathrm{K}$ monochromatic signals from each subarray pair location, a total of KMN measurements are required. On the other hand, for a pulsed waveform, just MN measurements would suffice. This scheme is, therefore, more time consuming. However, it is cost-effective since we do not need expensive equipment for pulse generation and processing. Also, the proposed scheme offers implementation flexibility because with just these $\mathrm{KMN}$ measurements, it is possible to form an image of the scene by synthesizing a variety of wideband waveforms such as sinc, raised cosine, etc. using appropriate Fourier coefficients $S\left(\omega_{k}\right)$ in eq. (7).

\section{EXPERIMENTAL RESULTS}

The synthetic aperture beamformer was set up in an anechoic chamber, using a single transmitter and a single receiver subarray to synthesize a 4-element transmit and an 8-element receive line array.

\subsection{Transmit and Receive Array Design}

Sensor placement is designed with the following physical constraints:

- Dimension of the anechoic chamber. This limits the combined span of transmit and receive arrays.

- The size of the antenna array element. This sets a minimum spacing at any time between the transmitter and the receiver.

Based on these constraints, we have designed the transmit and receive array apertures so as to achieve a spatial resolution of $0.43 \mathrm{~m}$ at a range of $6.7 \mathrm{~m}$. The array locations are given in Table 1. Figures 1(a) and (b) show the sum coarrays corresponding to $2 \mathrm{GHz}$ center frequency, and 1 $\mathrm{GHz}$ bandwidth, respectively. We can clearly see that the wideband coarray is richer than the single frequency one.

As our objective is to verify the working of the synthetic aperture beamformer, the wideband coarray was not used for optimal sensor placement. However, once incorporated, it would allow us to achieve the present resolution using fewer array element locations.

\subsection{System Parameters and Results}

A signal of $1 \mathrm{GHz}$ bandwidth with a center frequency of 2 $\mathrm{GHz}$ was chosen for imaging. HP-8510 network analyzer was used to synthesize this $1 \mathrm{GHz}$ signal, using 801 frequencies with a step size of $1.25 \mathrm{MHz}$. A conducting sphere of $12^{\prime \prime}$ diameter, at a range of $6.7 \mathrm{~m}$ and angle $0^{\circ}$, was used as the target. Two horn antennas, both with operational bandwidths from 1.0 to $12.4 \mathrm{GHz}$, were used as transmitter and receiver (See Fig. 2). Figure 3 shows the plan of the anechoic chamber.

Figure 4 shows the reconstructed received wideband pulses for each of the 32 transmit/receive subarray pairs. Note that the pulses are staggered in time because the delays encountered are different for each transmit/receive pair. Figure 5 shows the pulses time-aligned for boresight. In both these figures, the return immediately after the one from the sphere is due to the creeping wave phenomenon. The computed angular response of the synthetic aperture beamformer for a range of $6.7 \mathrm{~m}$ is plotted in Fig. 6 . This beampattern was obtained by applying unit weights to transmit and receive elements and using a uniform $S\left(\omega_{k}\right)$. The system clearly locates the spherical target at boresight.

\section{CONCLUSIONS}

We have successfully designed, implemented, and demonstrated a synthetic aperture beamformer for wideband imaging of near-field scenes using stepped frequency signal generation, coarray-based aperture synthesis, and post-data acquisition processing. The proposed beamformer design is particularly suitable for through-the-wall microwave imaging because of its low cost and ease of implementation.

\section{REFERENCES}

[1] R. T. Hoctor and S. A. Kassam, "The unifying role of the coarray in aperture synthesis for coherent and incoherent imaging", Proc. IEEE, vol. 78, no. 4, pp. 735-752, 1990.

[2] D. H. Johnson and D. G. Dudgeon, Array SignalProcessing, Prentice Hall, Englewood Cliffs, NJ, 1993.

[3] F. Ahmad and S. A. Kassam, "Coarray analysis of the wideband point spread function for active array imaging" Signal Processing, vol. 81, pp. 99-115, 2001.

[4] A. K. Luthra, S. A. Kassam, and R. B. Bernardi, "Body imaging using vectorial addition of acoustic reflection to achieve effect of scanning beam continuously focused in range", U.S. Patent No. 4,604,697, 1986.

[5] R. T. Hoctor and S. A. Kassam, "Array redundancy for active line arrays", IEEE Trans. on Image Processing, vol. 5, no. 7, pp. 1179-1183, 1996.

[6] F. Ahmad, et. al., "Aperture synthesis for a through-the-wall imaging system", Proc. ISSPIT, Dec. 2002.

[7] R. Benjamin, et. al., "Through-wall imaging using realaperture radar", Proc.URSI General Assembly, Aug., 2002.

[8] S. U. Pillai, Array Signal Processing, Springer-Verlag, New York, NY, 1989. 


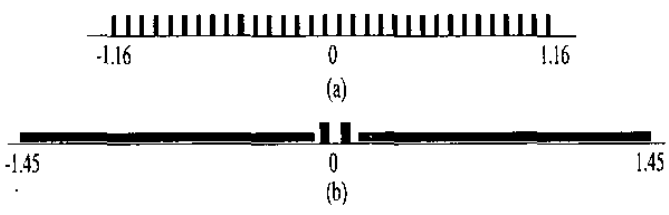

Figure 1: Sum Coarray corresponding to (a) $2 \mathrm{GHz}$; (b) 1 $\mathrm{GHz}$ bandwidth.

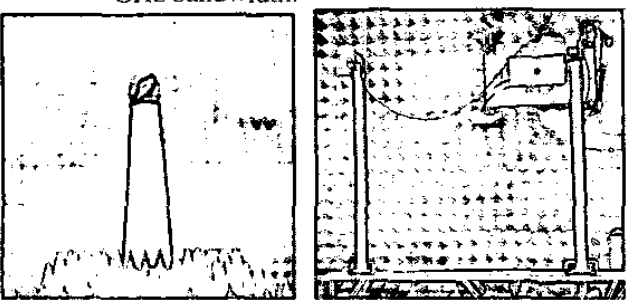

Figure 2: Target is shown on the left and the transmitter and receiver arrangement is shown on the right.

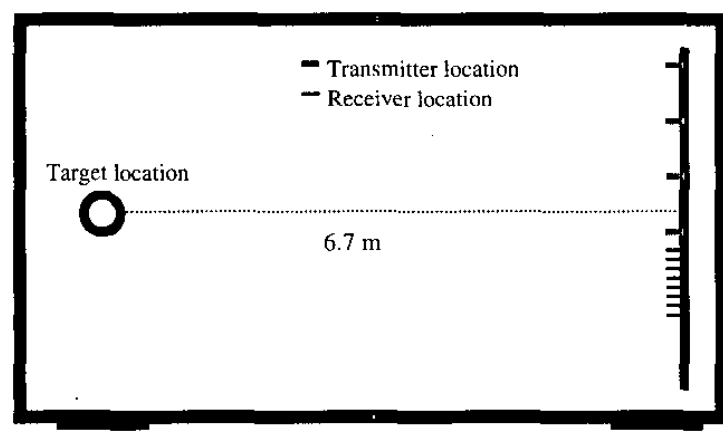

Figure 3: Plan diagram of the anechoic chamber

\begin{tabular}{|c|c|c|}
\hline No. & Transmitter Location $(\mathrm{m})$ & Receiver Location $(\mathrm{m})$ \\
\hline 1. & -1.6125 & 0.450 \\
\hline 2. & -1.0125 & 0.525 \\
\hline 3. & -0.4125 & 0.600 \\
\hline 4. & 0.1875 & 0.675 \\
\hline 5. & & 0.450 \\
\hline 6. & & 0.525 \\
\hline 7. & & 0.600 \\
\hline 8. & & 0.675 \\
\hline
\end{tabular}

Table 1: Transmit and Receive Array Locations

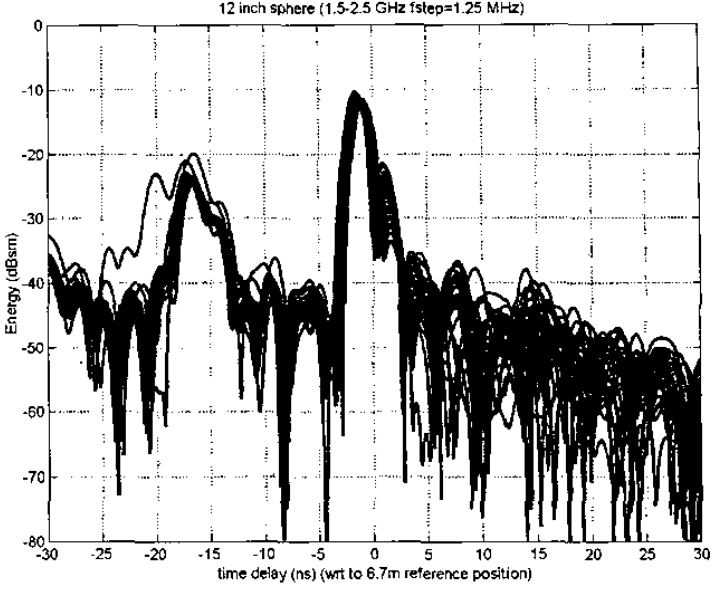

Figure 4: Staggered time pulses for 32 transmit/receive pairs

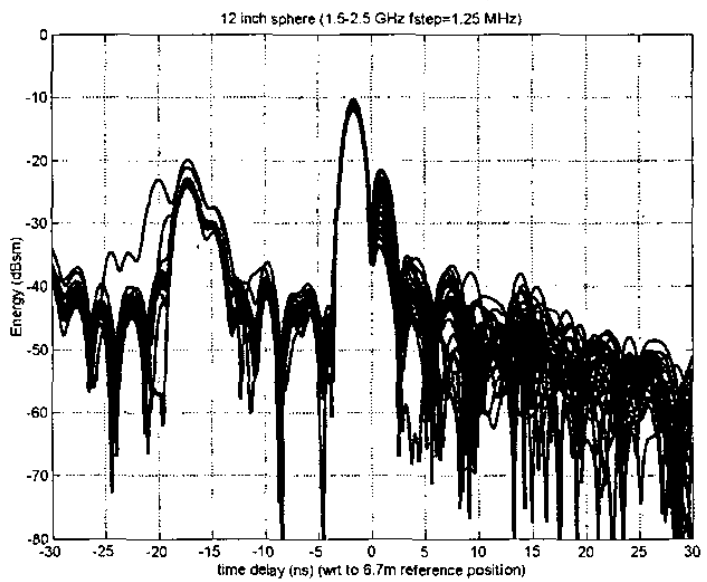

Figure 5: 32 Time pulses aligned for boresight

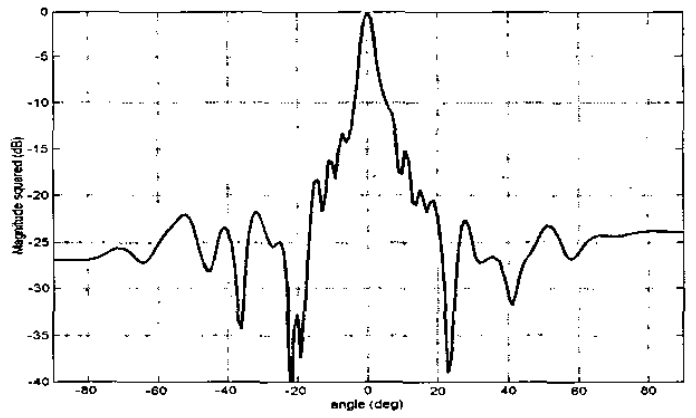

Figure 6: Angular response of the synthetic aperture beamformer 